\title{
Benzodiazepines: it's time to return to the evidence
}

Edward Silberman, Richard Balon, Vladan Starcevic, Richard Shader, Fiammetta Cosci, Giovanni A. Fava, Antonio E. Nardi, Carl Salzman and Nicoletta Sonino

\section{Summary}

We propose that discussions of benzodiazepines in the current psychiatric literature have become negatively biased and have strayed from the scientific evidence base. We advocate returning to the evidence in discussing benzodiazepines and adhering to clear definitions and conceptual rigour in commentary about them.

\section{Keywords}

Anti-anxiety drugs; anxiety disorders; comorbidity; drug interactions and side-effects; drugs of dependence.

\section{Copyright and usage}

(c) The Author(s), 2020. Published by Cambridge University Press on behalf of the Royal College of Psychiatrists..
Edward Silberman (pictured) is Professor of Psychiatry at Tufts University School of Medicine, Boston, USA, and a past President of the Association for Academic Psychiatry. Richard Balon is Professor of Psychiatry and Anesthesiology, Associate Chair and Programme Director in the Department of Psychiatry and Behavioral Neuroscience, Wayne State University, Detroit, USA. Vladan Starcevic is Associate Professor of Psychiatry at the University of Sydney, Australia. His professional and research interests include pharmacology of anxiety disorders and benzodiazepines. Richard Shader is Professor Emeritus of Integrative Physiology and Pathobiology at Tufts University, Boston, USA. He is Editor of the Journal of Clinical Psychopharmacology. Fiammetta Cosci is Associate Professor in Clinical Psychology at the University of Florence, Italy, and Associate Editor of Psychotherapy and Psychosomatics. Giovanni A. Fava is a senior scientist at the University of Padua, Italy, and Visiting Professor of Psychiatry, SUNY Buffalo, New York, USA. He is Editor-in-Chief of Psychotherapy and Psychosomatics. Antonio E. Nardi is Professor of Psychiatry at the Institute of Psychiatry, School of Medicine, Federal University of Rio de Janeiro, Brazil. He specialises in clinical psychopharmacology. Carl Salzman is Professor of Psychiatry at Harvard Medical School, Boston, USA. He was Chair of the American Psychiatric Association Task Force on Benzodiazepine Dependency and author of its report. Nicoletta Sonino is an endocrinologist, psychosomatic specialist and senior scientist at the University of Padova, Italy, and Visiting Professor at SUNY Buffalo, New York, USA.

When benzodiazepine anxiolytics were first introduced in the 1960s they were viewed as a liability-free alternative to barbiturates and meprobamate and were prescribed widely to patients with complaints of anxiety. After a decade of experience, it had become clear that benzodiazepines could be abused, and the pendulum began to swing towards suspicion of them. It is now commonly believed that they are dangerous drugs, prone to abuse and addiction. Treatment guidelines caution against their use as first-line or long-term therapy. It has become almost standard for clinical publications about benzodiazepines to issue warnings about dependence, abuse, addiction, tolerance or dangerousness, even when their central topic is an unrelated matter. Clinicians who advocate use of benzodiazepines may risk opprobrium from peers and institutions.

\section{Terminology}

The literature and diagnostic classifications such as the DSM and ICD use varying terminology when describing substance-use disorders. Here we differentiate between abuse (taking a drug to achieve an appetitive effect, or 'high') and misuse (any use that deviates from the way a medication has been prescribed).

\section{A reminder of what the evidence tells us}

The bulk of scientific literature on benzodiazepine safety, dependence and misuse tells a different story. Although demonstrating a range of potential liabilities, including cognitive and psychomotor impairment, possible risk in pregnancy and severe and/or prolonged withdrawal syndromes, it does not confirm that these medications are primary drugs of abuse or gateway drugs leading to other substance abuse. The database was scrutinised in the 1980s and 1990s in a series of extensive reviews, including a volume commissioned and published by the American Psychiatric Association. In aggregate, they comprise over 2000 literature citations, dealing with both animal and human studies bearing on abuse, misuse and dangerousness of benzodiazepines. ${ }^{1-3}$ Their authors conclude that benzodiazepines 'do not strongly reinforce their own use and are not widely abused drugs. When abuse does occur, it is almost always among persons who are also abusing alcohol, opiates or other sedative hypnotics' ${ }^{2}$ and that 'epidemiological studies of various populations of drug abusers have often found rates of nonmedical use of benzodiazepines that exceed those found in the general population [but] the preponderance of the extensive use of benzodiazepines is directed by physicians for disorders in which these drugs have proven therapeutic effect'. ${ }^{3}$ Although co-abuse of benzodiazepines has risen in the context of the opioid epidemic, there has been no newer evidence suggesting that either benzodiazepine abuse or any other substance abuse has its genesis in prescribed treatment for general (i.e. non-substanceabusing) patients. In his 2005 review of benzodiazepine abuse and dependence, O'Brien states, 'benzodiazepines are usually a secondary drug of abuse - used mainly to augment the high received from another drug or to offset the adverse effects of other drugs. Few cases of addiction arise from the legitimate use of benzodiazepines'. ${ }^{4}$ Although most of the literature on this topic is not recent, neither is it outdated; it is simply ignored.

\section{Reasons for the bias against benzodiazepines}

Why bias against a safe and useful class of medications has become so entrenched is not entirely clear and is itself a subject worthy of investigation. One factor may be that major pharmaceutical companies long ago abandoned benzodiazepines in favour of antidepressants, which we believe has had a substantial influence on practitioners and has left benzodiazepines with few people to speak up for them. Concern about co-abuse of benzodiazepines by opioid abusers, with potentially lethal consequences, may be another factor motivating physicians to avoid them. In this climate of opinion, discussions about benzodiazepines often blur important distinctions about their clinical pharmacology or 
describe them in inaccurately pejorative terms. We discuss below five instances of unfounded beliefs about benzodiazepines that we believe have been especially detrimental.

(a) Benzodiazepines prescribed for anxiety disorders are likely to be abused. Benzodiazepines have a short latency of onset to calming or sedating effects, which may make them attractive to people who abuse substances. However, they are not prone to being abused by those with no such history. Conflating risk in these two populations stigmatises people with anxiety disorders and deprives them of treatment that might restore them to more functional lives.

(b) Patients who misuse benzodiazepines are on a spectrum of drug abuse and are at risk of proceeding to frank abuse or addiction. Misuse is defined as any use of a medication that deviates from the way it has been prescribed by a clinician - including taking extra doses or taking less medication than prescribed. ${ }^{5}$ Addiction (called dependence in ICD-10) is defined by a cluster of behaviours that includes drug-taking to achieve appetitive effects (i.e. a high), preoccupation with the substance in question, temporary satiation, loss of control and persistent use despite negative consequences. Misuse of benzodiazepines is common, estimated at $17 \%$ of overall use. ${ }^{5}$ However, the great majority of people who deviate from doctors' prescriptions of them are trying to control symptomatic distress, not to get high, ${ }^{5}$ and there is no evidence that misuse is likely to lead to abuse. Abuse and addiction should be addressed by substance abuse treatment; misuse is a more heterogeneous phenomenon that may involve suboptimal prescribing, poor doctorpatient communication, and patients inappropriately attempting to eradicate all negative affect with medication. However, alarmed clinicians who automatically view patients who deviate from their instructions as medication abusers may demand that they taper off benzodiazepines, with detrimental consequences.

(c) Patients prescribed benzodiazepines tend to escalate their doses, which should preclude long-term use. There is a common belief that long-term benzodiazepine treatment is associated with tolerance to their anxiolytic effects and consequent dose escalation. The accumulated evidence is to the contrary: long-term treatment is associated with maintenance of therapeutic benefits, and no dose escalation. Tolerance does develop to the sedating and psychomotor effects of benzodiazepines, however. ${ }^{2}$ Failure to make this distinction may be the basis for withholding benzodiazepines, or for withdrawing them from patients who have been doing well on them during acute treatment.

(d) Benzodiazepines are dangerous in overdose. Benzodiazepines alone are among the safest of psychotropic medications, with lethal dose $\mathrm{LD}_{50}$ estimates for most in the range of thousands of $\mathrm{mg} / \mathrm{kg}$. Even alprazolam, which may be more toxic, has an estimated $\mathrm{LD}_{50}$ range of $300-2000 \mathrm{mg} / \mathrm{kg}$. Taken in conjunction with alcohol or opioids, they markedly raise the lethality of these already dangerous substances. That benzodiazepines are safe for the vast majority of people with anxiety disorders for whom they are prescribed is obscured by commonly used phrases such as 'benzodiazepine-related death' to describe a lethal combination of opioids and benzodiazepines ingested by a polysubstance-abusing person.

(e) Taking benzodiazepines long-term leads to dependence. The word 'dependence' almost invariably has pejorative connotation and may unfairly characterise patients when applied vaguely or inconsistently. As used in ICD-10, 'drug dependence' is essentially a syndrome of addiction. 'Dependence' may also be used to describe a physiological withdrawal syndrome, an entirely different phenomenon that occurs with many medications, and is not in itself a sign of addiction. ${ }^{4}$ People with anxiety disorders discontinuing chronic benzodiazepines may experience a syndrome that includes rebound anxiety, which clinicians may take as a reason to withhold long-term treatment. Dependence is commonly applied, without clear definition, to patients in long-term treatment with benzodiazepines who lack any of the behavioural characteristics of substance abuse. They may be labelled as dependent (or addicted or hooked) because of the potential for a withdrawal syndrome and told that they must deal with it by getting off their medication. It is not surprising that anxious patients summarily told that they are drug dependent and deprived of an effective medication have difficulty tapering off it. But their doctors may interpret their struggles as evidence that a benzodiazepine prescription was problematic to begin with.

\section{A call to clinicians}

It's time to return to the evidence about benzodiazepines and to conceptual rigour in interpreting it. Benzodiazepines are highly effective for treatment of anxiety disorders, but are not for everyone, have potential liabilities and are best used in conjunction with targeted psychotherapies. That polysubstance abuse often includes benzodiazepines, however, should not blind us to their appropriate use. Distinctions between abuse, addiction/dependence, misuse and physiological dependence may be challenging, but they are supported by the evidence and are clinically important. Conflating these phenomena will perpetuate stigma against benzodiazepines, the clinicians who prescribe them and the patients who take them. We invite colleagues to engage in evidence-based reappraisal of the benefits and risks of these medications and to abandon aspects of conventional wisdom that do not stand up to such scrutiny.

\section{Acknowledgement}

This manuscript arose out of discussions among the members of the International Taskforce on Benzodiazepines, an informal collaborative group of academic clinicians (including the authors) who are interested in disseminating accurate information about and fostering appropriate use of these medications.

Edward Silberman (D), MD, Tufts University School of Medicine Boston,

Massachusetts, USA; Richard Balon, MD, Department of Psychiatry and Behaviora Neuroscience, Wayne State University, Detroit, Michigan, USA; Vladan Starcevic, MD Nepean Clinical School, Faculty of Medicine and Health, University of Sydney, Australia; Richard Shader, MD, Graduate School of Biomedical Sciences, Tufts University, Boston, Massachusetts, USA; Fiammetta Cosci, MD, PhD, Department of Health Sciences, University of Florence, Italy; Giovanni A. Fava, MD, Department of Psychology,

University of Bologna; and Department of Psychiatry, State University of New York at University of Bologna; and Department of Psychiatry, State University of New York at
Buffalo, New York, USA; Antonio E. Nardi (D), MD, Institute of Psychiatry, School of Medicine, Federal University of Rio de Janeiro, Brazil; Carl Salzman, MD, Department of Psychiatry, Harvard Medical School, Boston, Massachusetts, USA; Nicoletta Sonino MD, Department of Medicine, University of Padua, Italy; and Department of Psychiatry, State University of New York at Buffalo, New York, USA

Correspondence: Edward Silberman. Email· esilberman@tuftsmedicalcenter org

First received 6 Feb 2020, final revision 27 Jul 2020, accepted 30 Aug 2020

\section{Author contributions}

E.S. prepared the first draft and subsequent revisions; all other authors reviewed the drafts, commented, made editorial suggestions, and reviewed and approved the final draft.

\section{Declaration of interest}

The authors are members of the International Taskforce on Benzodiazepines. C.Z. was Chair of the American Psychiatric Association Task Force on Benzodiazepine Dependency and author of its report.

ICMJE forms are in the supplementary material, available online at https://doi.org/10.1192/ bjp.2020.164. 


\section{References}

1 Woods JH, Katz JL, Winger G. Abuse liability of benzodiazepines. Pharmacol Rev 1987; 39: 251-413.

2 American Psychiatric Association Task Force on Benzodiazepine Dependency. Benzodiazepine Dependence, Toxicity, and Abuse: A Task Force Report of the American Psychiatric Association. APA, 1990.
3 Woods JH, Katz JL, Winger G. Benzodiazepines: Use, abuse, and consequences. Pharmacol Rev 1992; 44: 151-347.

4 O'Brien CP. Benzodiazepine use, abuse, and dependence. J Clin Psychiatry 2005; 66(suppl 2): 28-33.

5 Maust DT, Lin LL, Blow FC. Benzodiazepine use and misuse among adults in the United States. Psych Services 2019; 70: 97-106.

\section{psychiatry} in theatre

\section{Soviet theatre in the fight against neurasthenia in the 1920s and 1930 s}

\section{Igor J. Polianski and Oxana Kosenko (iD}

Neurasthenia was first defined by the renowned New York neurologist George Beard (1839-1883) in 1869. He described it as a lack of nerve force that developed due to the stress of modern society. Since neurasthenia had been perceived as a side-effect of a modernising America, Beard called it 'the American disease'. However, it seemed to spread rapidly to Europe. In 1887, the Russian psychiatrist Pavel Kovalevsky (1849-1931) even proclaimed neurasthenia a 'Russian disease', given the large number of 'neurasthenics' in his country. After the Russian Revolution, in the 1920s, neurasthenia was considered one of the most widespread mental disorders among the ranks of the new political and executive elites. Soviet psychiatrists believed that the cause of the nervous exhaustion lay in the revolutionary sufferings of Bolsheviks during the civil war and in psychological stress during the post-war reconstruction of the country. The work-related emotional overstraining often led to suicide. In 1925, the suicide rate among Communist Party activists reached $12.5 \%$ of all deaths. Neurasthenia was also regarded as a common illness of intellectuals.

It is therefore not surprising that neurasthenia came into the repertoire of the Moscow Theatre for Sanitary Culture in the 1920s. The theatre was founded in 1924 for purposes of hygiene propaganda. Its plays were devoted to many burning social and health problems, such as prostitution, alcoholism, protection of mothers and children, and sexually transmitted, infectious and, last but not least, nervous diseases. 'Psycho-hygienic' plays about the dangers of overworking and overtiredness had to agitate for mental health. One such play, the comedy Nerve Dance (1928) by Aleksandr Narodetsky (1895-?) and Mark Triger (1896-1941), shows a former civil war hero and director of a sugar refinery Vasily Terentyev, who works restlessly day and night for its sustainable future. Being under permanent stress and overloaded with work he turns into a neurotic person who is no longer able to control his emotions and acts like a 'hysterical woman'. Unexpectedly, he is appointed interim director of a factory in a small town. His new colleagues and young communists open his eyes to his neurasthenia by putting on a music show in which his behaviour is caricatured. Three months later, he returns to his hometown fully recovered from his illness.

A radical change in Soviet politics in 1929, known as the 'great break', marked the beginning of the forced industrialisation in the USSR. From then on, the country was racked by socialist emulations and overfulfilment of production plans. In accordance with the new political course, Soviet occupational physicians were forced to declare that motivated work in a socialist country had a vitalising effect on the entire organism. Thus, in the 1930s, fictional neurasthenics in the psycho-hygienic plays were replaced by hypochondriacs. Both adaptations of well-known classics such as The Imaginary Invalid by Molière (Fig. 1) and new socialist plays such as The Suicide by Dmitri Dolev (1883-1944) and Nikolai Mertsalsky (1896-1964) had to ridicule sickness as an imaginary construct of a work-shy hypochondriac.

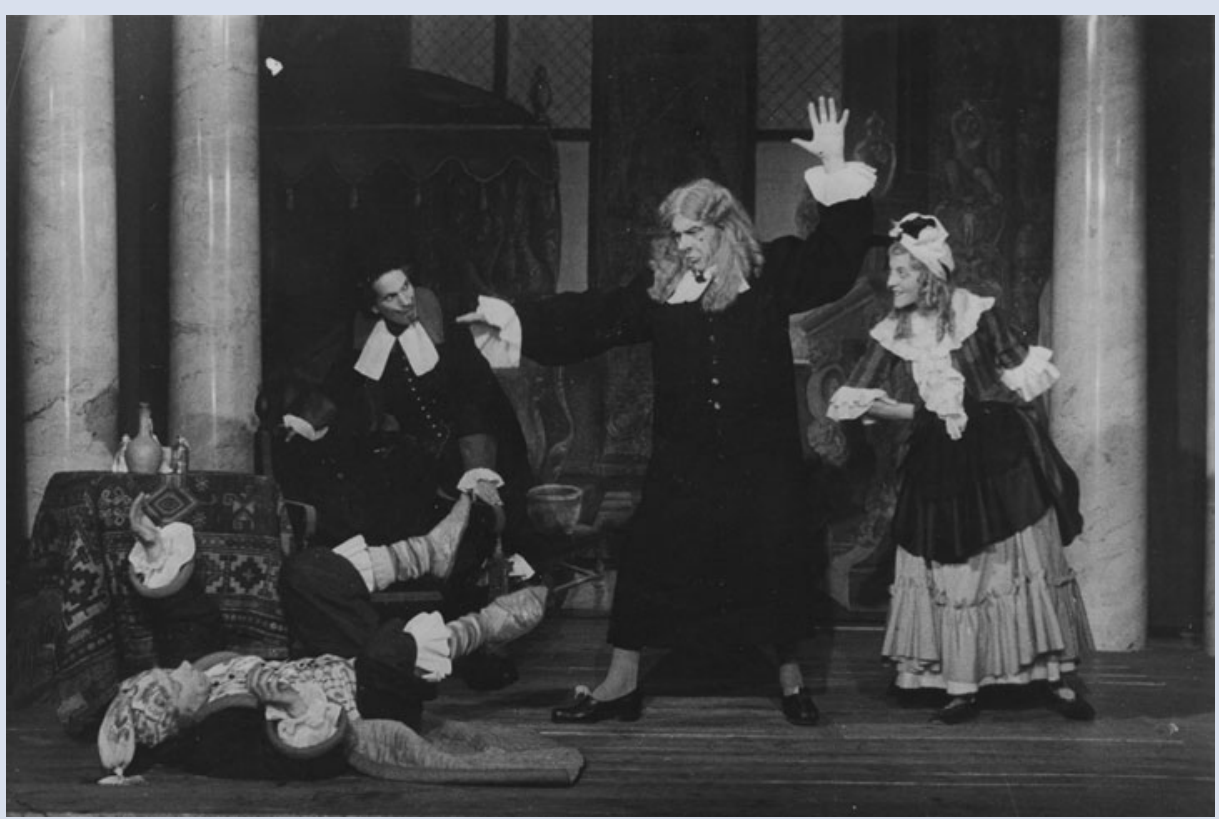

Fig. 1 Scene from Molière's The Imaginary Invalid (1929) at the Moscow Theatre for Sanitary Culture, 1944. Reproduced with permission from the Russian State Archive of Scientific-Technical Documentation, 178/5/110, p. 13. 